\title{
Precursor B cell lymphoid blast crisis of chronic myeloid leukemia with novel chromosomal abnormalities: A case report
}

\author{
HAMOUD AL-KHALLAF $^{1}$, HANI ALALI $^{1}$ and ADIL ALKHATTI ${ }^{2}$ \\ ${ }^{1}$ Department of Pathology and Laboratory Medicine, King Fahad Specialist Hospital, Dammam 32253; \\ ${ }^{2}$ Cancer Institute, Johns Hopkins Aramco Healthcare, Dhahran 34465, Saudi Arabia
}

Received November 24, 2017; Accepted March 19, 2018

DOI: $10.3892 / \mathrm{ol} .2018 .9497$

\begin{abstract}
Chronic myeloid leukemia (CML) is a clonal hematopoietic stem cell disorder. It is characterized by the presence of the Philadelphia $(\mathrm{Ph})$ chromosome, $\mathrm{t}(9 ; 22)(\mathrm{q} 34.1 ; \mathrm{q} 11.2)$, which carries the $B C R-A B L 1$ fusion gene. Tyrosine kinase inhibitors (TKIs) have markedly changed the treatment approach of CML and have become the first-line agents for almost all CML patients. However, certain patients experience resistance to these medications, which occurs through several mechanisms, including the accumulation of TKI-resistant chromosomal abnormalities. The present study reports a case of a 27-year-old Saudi male with CML receiving TKI treatment, who presented with precursor B-cell lymphoblastic crisis demonstrating the presence of the novel combined chromosomal abnormalities; non-Ph der(22), i(9) and $\operatorname{der}(20)$, carrying the $B C R-A B L 1$ fusion gene. This case report adds to the literature on novel TKI-resistance-conferring chromosomal abnormalities and links them to precursor B-cell lymphoblastic crisis.
\end{abstract}

Correspondence to: Dr Hamoud Al-Khallaf or Mr. Hani Alali, Department of Pathology and Laboratory Medicine, King Fahad Specialist Hospital, 6830 Ammar Bin Thabit Street, Al Muraikabat, Dammam 32253, Saudi Arabia

E-mail:drhhk@hotmail.com

E-mail: HaniM.Ali@kfsh.med.sa

Abbreviations: CML, chronic myeloid leukemia; $\mathrm{Ph}$, Philadelphia; TK, tyrosine kinase; TKI, tyrosine kinase inhibitor; BCR, breakpoint cluster region gene; ABL1, c-Abelson gene; WBC, white blood cell; CBC, complete blood count; CD, cluster of differentiation; FITC, fluorescein isothiocyanate; PE, phycoerythrin; APC, allophycocyanin; PerCP, peridinin chlorophyll; TdT, terminal deoxynucleotidyl transferase; MPO, myeloperoxidase; FISH, fluorescence in situ hybridization; RT-qPCR, reverse transcription-quantitative polymerase chain reaction; $\mathrm{Hb}$, hemoglobin; IS, International Scale; ALL, acute lymphocytic leukemia

Key words: chronic myeloid leukemia, precursor B cell, blast crisis, tyrosine kinase inhibitor, cytogenetic abnormalities

\section{Introduction}

CML is a myeloproliferative disorder that is characterized by the presence of the reciprocal translocation $\mathrm{t}(9 ; 22)$, which forms the Philadelphia $(\mathrm{Ph})$ chromosome. During this translocation, the breakpoint cluster region $(B C R)$ gene at position $22 \mathrm{q} 11.2$ is juxtaposed to the c-Abelson $(A B L 1)$ gene at 9q34.1, forming the $B C R-A B L 1$ fusion gene, which encodes a constitutively active tyrosine kinase $(\mathrm{TK})$ protein $(1,2)$. The constitutively active protein is associated with increased levels of erythrocytes, monocytes, megakaryocytes, myelocytes and platelets in the peripheral blood and marked myeloid hyperplasia in the bone marrow (3).

CML presents in one of three phases: Chronic phase, accelerated phase or blast crisis. The latter is of myeloid, lymphoid or mixed-lineage phenotype (4).

TKIs have markedly changed the approach to CML management. TKIs have improved patient outcomes to the extent that they are now currently accepted as the first-line agents for nearly all patients with CML, regardless of the phase of the disease. However, certain patients experience resistance to these medications; this occurs through several mechanisms including the accumulation of additional cytogenetic abnormalities, which can confer a survival advantage to the treated myeloid cells. The most common cytogenetic abnormalities include an additional $\mathrm{Ph}$ chromosome, trisomy 8 and isochromosome $17 \mathrm{q}(5,6)$. Several other less common cytogenetic abnormalities have been reported; however, to the best of our knowledge, those found in the present case have not been previously reported.

\section{Case report}

Presentation. The present case involves a 27-year-old Saudi male patient, whose initial presentation was three years prior. At that time, he presented with pallor and abdominal distension. He was revealed to have significant splenomegaly and marked leukocytosis, with a white blood cell (WBC) count of $105 \times 10^{9} / 1$. Subsequent investigations confirmed the diagnosis of CML. The patient was initially treated with imatinib; however, due to myelosuppression, the treatment was changed to dasatinib. Subsequently, due to a skin reaction, the treatment was changed to nilotinib (100 mg/day), which the patient clinically responded to and tolerated well. 
At his current presentation, the patient had fever, bone pain and cytopenia. Investigations confirmed the diagnosis of precursor B cell acute lymphoblastic leukemia with the presence of the novel combined chromosomal abnormalities of non-Ph der(22), i(9), and $\operatorname{der}(20)$, carrying the BCR-ABLI fusion gene.

Complete blood count $(C B C)$. CBC with differential was performed using an Automatic Hematological Analyzer Sysmex XE-5000 (Sysmex America, Inc., Lincolnshire, IL, USA).

Immunophenotyping. Immunophenotyping was performed on the patient's bone marrow aspirate as follows; upon collection of the bone marrow aspirate, $0.5 \mathrm{ml}$ of the sample was mixed with $10 \mathrm{ml}$ of red blood cell lysing solution and centrifuged at $540 \mathrm{x} \mathrm{g}$ for $5 \mathrm{~min}$. The supernatant was discarded and the cell pellet further washed with PBS. A $100 \mu 1$ aliquot of cell suspension with an adjusted concentration of $10 \times 10^{9}$ cells/ 1 was added to tubes containing commercial pre-titrated volumes of labelled monoclonal antibody cocktails to bind several antigens, surface and cytoplasmic clusters of differentiation (CD) (BD Biosciences, San Jose, CA, USA) and incubated in the dark for $15 \mathrm{~min}$ at room temperature. These monoclonal antibodies were used in conjunction with four fluorochromes [fluorescein isothiocyanate (FITC), phycoerythrin (PE), allophycocyanin (APC) and peridinin chlorophyll (PerCP)] in each tube where they were diluted by factor of 20. FITC labelled antibodies bound to CD14 (cat. no. 561712), surface and cytoplasmic IgG (cat. no. 560952), surface and cytoplasmic CD3 (cat. no. 561806), CD34 (cat. no. 560942), cytoplasmic CD66 (cat. no. 551479), IgM (cat. no. 562029), CD33 (cat. no. 561818), CD38 (cat. no. 560982), CD2 (cat. no. 561759), CD64 (cat. no. 560970), terminal deoxynucleotidyl transferase (TdT) (cat. no. 347194), CD45 (cat. no. 560976) and (myeloperoxidase) MPO (cat. no. 340580). PE labelled antibodies bound to surface and cytoplasmic IgG (cat. no. 560951), cytoplasmic CD11 (cat. no. 560999), CD8 (cat. no. 560959), CD10 (cat. no. 561002), CD56 (cat. no. 561903), CD117 (cat. no. 561682), CD13 (cat. no. 560998), CD7 (cat. no. 561934), CD58 (cat. no. 560959) and cytoplasmic CD79a (cat. no. 555935). APC labelled antibodies bound surface and cytoplasmic IgG (cat. no. 562025), CD20 (cat. no. 560900), CD4 (cat. no. 561840), CD19 (cat. no. 561742), CD15 (cat. no. 561716), CD22 (cat. no. 562860), CD11b (cat. no. 340937), CD5 (cat. no. 340583), HLA-DR (cat. no. 560896), cytoplasmic CD22 (cat. no. 562860), CD25 (cat. no. 340939) and CD34 (cat. no. 560940). PerCP labelled antibodies bound CD45 (cat. no. 561086) and cytoplasmic CD3 (cat. no. 347344).

For intracellular staining (i.e., for CD79a, CD3, TdT and MPO), lymphocyte permeabilization preceded the addition of cytoplasmic and nuclear antibodies. This was achieved by adding $0.5 \mathrm{ml}$ FACS Permeabilizing Solution to $100 \mu \mathrm{l}$ of the lysed sample followed by 10-minute dark incubation at room temperature. The FACS Permeabilizing Solution was prepared by diluting $1 \mathrm{ml}$ of BD Permeabilizing stock solution (cat. no. 340973; BD Biosciences) in $9 \mathrm{ml}$ of distilled water.

The samples were processed with a FACSCanto II cell analyzer and the analysis was performed using FACS Diva
Software (version 8.0.1; BD Biosciences). The flow cytometry data was analyzed with a threshold of 25,000 events. For gating, forward scatter, side scatter, CD45, CD3, CD19 in addition to other lineage specific markers were used. The lineages of the blasts were determined in each case depending upon the expression of lineage-specific markers where an expression for a certain marker was considered positive if the percentage of the cells expressing that marker was $\geq 20 \%$ and negative if $<20 \%$, except for TdT and MPO where the threshold was $10 \%$ as recommended (7).

Cytogenetic analysis. Chromosomal analysis using GTG banding was performed as described previously (8). Karyotyping was performed in 20 metaphases from the patient's unstimulated bone marrow sample according to the nomenclature of the International System for Human Cytogenetics (9).

Fluorescence in situ hybridization (FISH). FISH was performed using Vysis $B C R-A B L 1$ Tri-Color Dual-Fusion FISH Probes (Abbott Pharmaceutical Co. Ltd., Lake Bluff, IL, USA) to detect the $B C R-A B L 1$ translocation, as described previously (10).

Molecular analysis. EDTA whole blood samples from the patient were used for quantification of the BCR-ABL1 P210 transcript by reverse transcription-quantitative polymerase chain reaction (RT-qPCR). This was performed using the GeneXpert ${ }^{\circledR}$ Dx System (Roche Diagnostics GmbH, Mannheim, Germany) as described previously (11).

Initial presentation. At initial presentation, the patient's $\mathrm{CBC}$ with differential revealed a hemoglobin $(\mathrm{Hb})$ level of $8.6 \mathrm{~g} / \mathrm{dl}$, a platelet count of $188 \times 10^{9} / 1$ and a WBC count of $105 \times 10^{9} / 1$, comprised of $52 \%$ neutrophils, $1 \%$ lymphocytes, $3 \%$ monocytes, $0 \%$ eosinophils, $0 \%$ basophils, $1 \%$ promyelocytes, $2 \%$ myelocytes and $5 \%$ blast cells. The patient's peripheral blood smear revealed normocytic hypochromic anemia with anisocytosis and schistocytosis. There was marked leukocytosis with a significant number of immature myeloid precursors, indicating leukoerythroblastosis. The patient's bone marrow aspirate was hemodiluted, but revealed moderate cellularity with the presence of myeloid, erythroid and megakaryocytic lineages and $\sim 5 \%$ blast cells. The karyotype of each of the 10 metaphases obtained from the bone marrow aspirate was 46,XY,t(9;22)(q34.1;q11.2). FISH analysis confirmed the presence of the $B C R-A B L 1$ fusion in $95 \%$ of nuclei in the aspirate. Molecular studies of the patient's peripheral blood determined a $B C R-A B L 1$ fusion transcript level of $100 \%$ International Scale (IS) units. Based on these results, the patient was diagnosed with CML.

Current status. At the current presentation, the patient's CBC with differential revealed an $\mathrm{Hb}$ level of $10.5 \mathrm{~g} / \mathrm{dl}$, a platelet count of $37 \times 10^{9} / 1$ and a WBC count of $0.9 \times 10^{9} / 1$, comprised of $48 \%$ neutrophils, $25 \%$ lymphocytes, $6 \%$ variant lymphocytes, $2 \%$ monocytes, $0 \%$ eosinophils, $0 \%$ basophils and $14 \%$ blast cells. The peripheral blood smear revealed pancy topenia with the presence of blasts. 

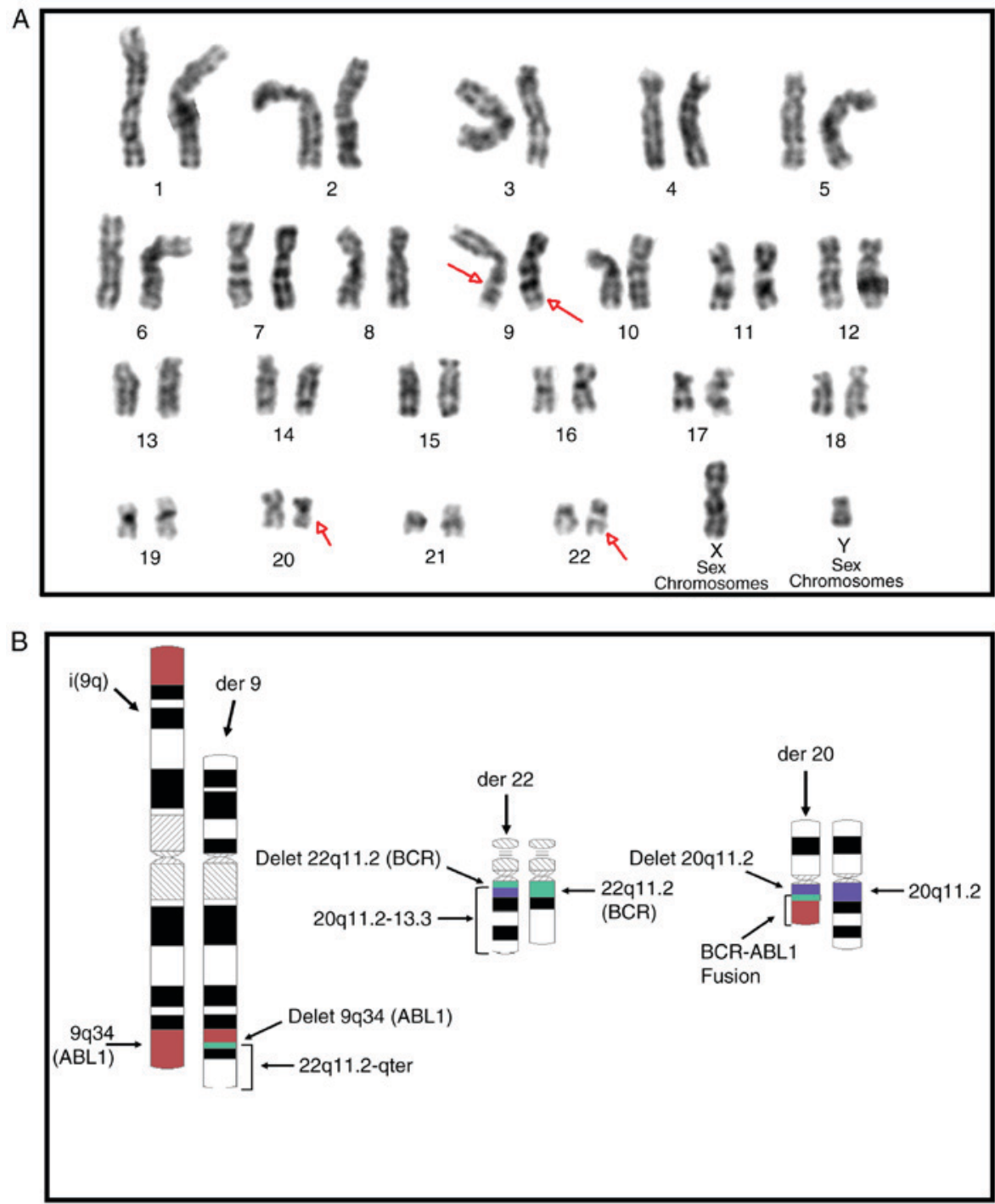

Figure 1. (A) Giemsa-banding karyogram of the patient's bone marrow. Isochromosome 9q, derivative chromosomes 20 and 22 (red arrows) are presented, where the extra material on 20 containing $B C R-A B L 1$ fusion is derived from chromosome 22. (B) Ideogram of all chromosomal abnormalities observed in this case with breakpoints marked.
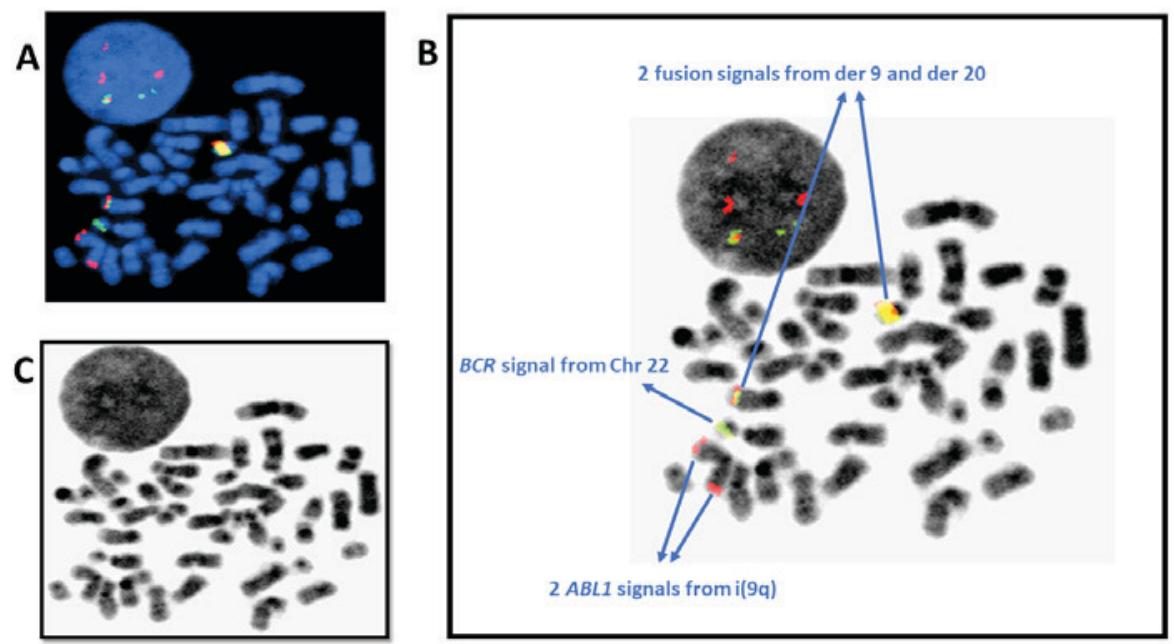

Figure 2. FISH analysis of one metaphase and one interphase nucleus. (A) Showing two $A B L 1$ (red) signals, one $B C R$ signal (green), and two fusion gene $B C R-A B L 1$ signals (yellow). (B) Labelled inverted image showing two $A B L 1$ signals on the two long arms of isochromosome 9 (red), one $B C R$ signal (green) on chromosome 22, and two fusion gene $B C R-A B L 1$ signals (yellow) one on the long arm of der 9 and one on that of der 20, confirming the abnormalities seen on the karyogram. (C) Non-labelled inverted image identifying the chromosomes containing the signals. 

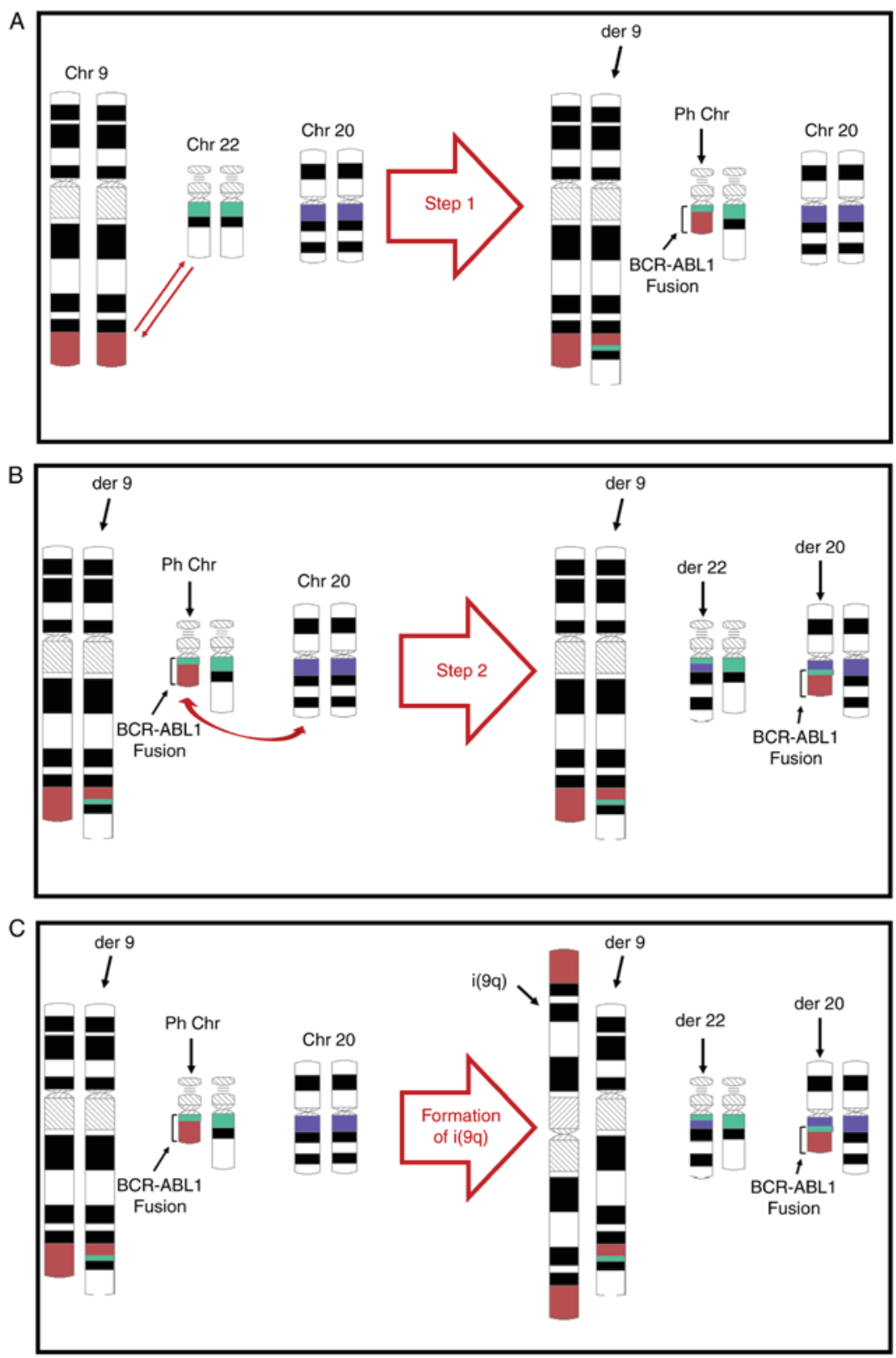

Figure 3. Ideogram illustrating the formation of the cytogenetic abnormalities observed in this case. (A) Ideogram of the proposed first step where a reciprocal translocation between chromosomes 9 and 22 (red arrows) formed the Philadelphia $(\mathrm{Ph})$ and derivative (der) 9 chromosomes. (B) Ideogram of the proposed second step where a reciprocal translocation (bidirectional red arrow) between chromosome 20 and Ph chromosome formed der(22) and der(20) chromosomes (the latter carrying the $B C R-A B L 1$ fusion gene). (C) Isochromosome $9 \mathrm{q}[\mathrm{i}(9 \mathrm{q})]$ formation as an additional cytogenetic abnormality that did not necessarily occur in this order.

The bone marrow aspirate revealed markedly increased blasts with markedly decreased myeloid, erythroid and megakaryocytic cell lineages. Immunophenotyping analysis of the aspirate revealed that the blast cells were positive for CD34 (partial, i.e., only a subset of the population of interest was positive), cytoplasmic CD79a, CD19, CD10, cytoplasmic TdT, CD20, HLA-DR, CD73 (partial), CD58, CD44, CD200, CD24, cytoplasmic CD66 and CD72 antigens. The cells were negative for cytoplasmic myeloperoxidase, cytoplasmic CD3, surface CD3, CD7, surface IgM, CD45 (negative to low), CD117, cytoplasmic CD22, CD33, CD13, CD38, CD123 and CD86 antigens, consistent with the precursor B-cell lymphoblastic nature of these blast cells.

A bone marrow biopsy revealed $99 \%$ cellularity with 95-99\% blasts. The number of morphologically normal myeloid, erythroid and megakaryocytic cells was markedly decreased to absent. Karyotyping performed on 20 metaphases from the bone marrow revealed the following: 46,XY,i(9)(q10),der(22)t(9;22) (q34.1;q11.2)t(20;22)(q11.2;q11.2)[18]/46,XY[2](Fig. 1A). This result, combined with that of the FISH analysis, confirmed the presence of a clone with the concurrent cytogenetic abnormalities of $\mathrm{i}(9)(\mathrm{q} 10)$, non-Philadelphia $\operatorname{der}(22)$ and $\operatorname{der}(20)$ carrying the $B C R-A B L 1$ fusion gene (Figs. $1 \mathrm{~B}$ and $2 \mathrm{~A}-\mathrm{C}$ ).

\section{Discussion}

The blast crisis demonstrated in this case was of the precursor B cell lymphoblastic type. It is well established that $230 \%$ of blast crises in CML are of the lymphoid rather than the 
myeloid phenotype. The aberrant cytogenetic abnormalities observed in this case, in addition to the $B C R-A B L 1$ fusion during $\mathrm{Ph}$ chromosome formation, were $\mathrm{i}(9 \mathrm{q})(\mathrm{q} 10)$ formation and the reciprocal translocation between the $\mathrm{Ph}$ chromosome and 20q11.2. To the best of our knowledge, this is the first case reported to combine these cytogenetic aberrations. This case report also links these cytogenetic aberrations to the precursor B cell lymphoblast phenotype. Ascertaining the blast phenotype has its own therapeutic implications, since the treatment protocol of lymphoid blast crisis is different to that of the myeloid type. In other words, linking these abnormalities to lymphoid crisis, in this case, had therapeutic implications since this directed the treatment toward vincristine- and prednisone-based protocols (i.e., lymphoid blast crisis protocol).

From the patient's history, initial karyotyping and FISH results, it may be concluded that these cytogenetic abnormalities did not occur simultaneously. The first abnormality was the classical $\mathrm{t}(9 ; 22)(\mathrm{q} 34.1 ; \mathrm{q} 11.2)$, resulting in Ph chromosome formation (Fig. 3A). The other two abnormalities were the i(9)(q10) formation and the reciprocal translocation between $\mathrm{Ph}$ and 22 chromosomes, transferring the $B C R-A B L 1$ fusion gene to the latter (Fig. 3B and C). These two events must have followed the $\mathrm{Ph}$ chromosome formation during the clonal evolution that conferred TKI resistance. It is not possible to tell whether these two latter events occurred simultaneously, or their order of occurrence if they did occur sequentially. For demonstration purposes only, i(9) (q10) formation is indicated in Fig. 3C to be the final event.

It is likely that the B cell lymphoid nature of the blast crisis observed in this patient is attributed to the loss of $9 p$ during $i(9 q)$ formation. This possibility stems from the fact that $9 p$ loss is a known recurrent cytogenetic abnormality observed in chronic lymphocytic leukemia and mantle cell lymphoma (12). This arm contains genes that are important for $\mathrm{B}$ cell differentiation and cell cycle regulation, including $P A X 5$ and $C D K N 2 A$. The paired box gene $P A X 5$ was altered in $32 \%$ of precursor B cell acute lymphocytic leukemia (ALL) cases (13). In Ph-positive-ALL patients, an additional 9p abnormality [including $\mathrm{i}(9)(\mathrm{q} 10)$ ] had a negative impact on disease-free survival (14-16). This predicts a poor prognosis for the patient discussed in the present study; however, extended follow-up is required to confirm this.

Due to patient being $<40$ years old, they were subjected to a pediatric $\mathrm{Ph}$-positive-ALL treatment protocol, as recommended (17). He received imatinib (400 mg daily) and 4 drugs as induction therapy: Daunorubicin $\left(25 \mathrm{mg} / \mathrm{m}^{2}\right.$ weekly for 4 doses), vincristine [2 $\mathrm{mg} /$ week intravenously for 4 doses], prednisone $\left(60 \mathrm{mg} / \mathrm{m}^{2}\right.$ for 28 days $)$ and asparaginase $\left(2,500 \mathrm{U} / \mathrm{m}^{2}\right.$ on day 6$)$. He also received intrathecal central nervous system prophylaxis chemotherapy with cytarabine on day 1 and methotrexate on days 8 and 29. A complete remission was achieved on day 29 , which was confirmed by flow

cytometry, cytogenetic and molecular testing results, with minimal residual disease $(<0.1 \%)$ and undetectable levels of $B C R-A B L 1$ mRNA. The patient then continued to receive $400 \mathrm{mg}$ imatinib daily.

Consolidation therapy commenced on day 36 of induction. Phase one consisted of etoposide $\left(100 \mathrm{mg} / \mathrm{m}^{2}\right)$ and ifosfamide $\left(1,800 \mathrm{mg} / \mathrm{m}^{2}\right)$, daily for 5 days. Intrathecal therapy was administered on days 8 and 15 of consolidation therapy, and consisted of methotrexate $(15 \mathrm{mg})$, hydrocortisone $(15 \mathrm{mg})$ and cytarabine (30 mg). Phase two of consolidation consisted of high-dose methotrexate $\left(5,000 \mathrm{mg} / \mathrm{m}^{2}\right)$ on day 1 and high-dose cytarabine $\left(3,000 \mathrm{mg} / \mathrm{m}^{2}\right)$ every $12 \mathrm{~h}$ on days 2 and 3 ( 4 doses in total). Triple intrathecal therapy was administered on day 1 . The patient has recently undergone tissue-matched stem cell transplantation.

In conclusion, the present study reports a case of TKI-resistant Ph-positive-CML presenting with lymphoblastic crisis wherein the blast cells, in addition to the $\mathrm{Ph}$ chromosome, exhibited additional novel combined cytogenetic abnormalities. This report adds to the literature on newly identified TKI-resistance-conferring cytogenetic abnormalities, and also links them to precursor B cell lymphoblastic crisis. This also has its own therapeutic implications since the blast phenotype determines the treatment protocol.

\section{Acknowledgements}

The authors would like to thank Ms. Amal Al-Khattaf (Flow Cytometry Section, King Fahad Specialist Hospital) for her assistance.

\section{Funding}

No funding was received.

\section{Availability of data and materials}

The datasets used and/or analyzed during the current study are available from the corresponding author on reasonable request.

\section{Authors' contributions}

HAK wrote the manuscript, drew the figures, designed the study, interpreted the data, contributed to addressing all questions related to the accuracy and integrity of this study, and given his final approval of the version to be published. HA initiated this research project and interpreted the cytogenetic results. AA followed up the patient and provided the test results. All authors read and approved the final manuscript.

\section{Ethics approval}

Approval from Johns Hopkins Aramco Healthcare (JHAH) Institutional Review Board and Ethics Committee was obtained to publish this case report.

\section{Patient consent for publication}

Written informed consent was obtained from the patient for publication of this case report and any accompanying figures.

\section{Competing interests}

The authors declare that they have no competing interests.

\section{References}

1. Kuru D, Tarkan Argüden Y, Ar M, Çırakoğlu A, Öngören Ş, Yılmaz Ş, Eşkazan A, Deviren A, Soysal T, Hacıhanefioğlu S and Ülkü B: Variant Philadelphia translocations with different breakpoints in six chronic myeloid leukemia patients. Turk J Haematol 28: 186-192, 2011. 
2. Aliano S, Cirmena G, Fugazza G, Bruzzone R, Palermo C and Sessarego M: Standard and variant Philadelphia translocation in a CML patient with different sensitivity to imatinib therapy. Leuk Res Rep 2: 75-78, 2013.

3. Sawyers CL: Chronic myeloid leukemia. N Engl J Med 17: 1330-1340, 1999.

4. Cervantes F, Villamor N, Esteve J, Montoto S, Rives S, Rozman C and Montserrat E: 'Lymphoid' blast crisis of chronic myeloid leukaemia is associated with distinct clinicohaematological features. Br J Haematol 100: 123-128, 1998.

5. Milojkovic D and Apperley J: Mechanisms of resistance to imatinib and second-generation tyrosine inhibitors in chronic myeloid leukemia. Clin Cancer Res 15: 7519-7527, 2009.

6. Johansson B, Fioretos T and Mitelman F: Cytogenetic and molecular genetic evolution of chronic myeloid leukemia. Acta Haematol 107: 76-94, 2002.

7. Abdulsalam AH, Nadal-Melsio $E$ and Naresh KN: Complementarity of evaluation of myeloperoxidase expression by flow cytometry and immunohistochemistry on bone marrow trephine biopsy sections in acute myeloid leukemia. Cytometry B Clin Cytom 86: 70-73, 2014.

8. Claussen U, Michel S, Mühlig P, Westermann M, Grummt UW, Kromeyer-Hauschild K and Liehr T: Demystifying chromosome preparation and the implications for the concept of chromosome condensation during mitosis. Cytogenet Genome Res 98: 136-146, 2002

9. Simons A, Shaffer LG and Hastings RJ: Cytogenetic nomenclature: Changes in the ISCN 2013 compared to the 2009 edition. Cytogenet Genome Res 141: 1-6, 2013.

10. Siu LL, Ma ES, Wong WS, Chan MH and Wong KF: Application of tri-colour, dual fusion fluorescence in situ hybridization (FISH) system for the characterization of BCR-ABL1 fusion in chronic myelogenous leukaemia (CML) and residual disease monitoring. BMC Blood Disord 9: 4, 2009.
11. Jobbagy Z, van Atta R, Murphy KM, Eshleman JR and Gocke CD: Evaluation of the Cepheid GeneXpert BCR-ABL assay. J Mol Diagn 9: 220-227, 2007.

12. Beà S, López-Guillermo A, Ribas M, Puig X, Pinyol M, Carrió A, Zamora L, Soler F, Bosch F, Stilgenbauer S, et al: Genetic imbalances in progressed B-cell chronic lymphocytic leukemia and transformed large-cell lymphoma (Richter's syndrome). Am J Pathol 161: 957-968, 2002.

13. Mullighan CG, Goorha S, Radtke I, Miller CB, Coustan-Smith E, Dalton JD, Girtman K, Mathew S, Ma J, Pounds SB, et al: Genome-wide analysis of genetic alterations in acute lymphoblastic leukaemia. Nature 446: 758-764, 2007.

14. Haidar MA, Cao XB, Manshouri T, Chan LL, Glassman A, Kantarjian HM, Keating MJ, Beran MS and Albitar M: p16INK4A and p15INK4B gene deletions in primary leukemias. Blood 86: 311-315, 1995.

15. Mrózek K, Harper DP and Aplan PD: Cytogenetics and molecular genetics of acute lymphoblastic leukemia. Hematol Oncol Clin North Am 23: 991-1010, 2009.

16. Yanada M, Takeuchi J, Sugiura I, Akiyama H, Usui N, Yagasaki F, Kobayashi T, Ueda Y, Takeuchi M, Miyawaki S, et al: Karyotype at diagnosis is the major prognostic factor predicting relapse-free survival for patients with Philadelphia chromosome-positive acute lymphoblastic leukemia treated with imatinib-combined chemotherapy. Haematologica 93: 287-290, 2008.

17. Kliman D, Barnett M, Broady R, Forrest D, Gerrie A, Hogge D, Nantel S, Narayanan S, Nevill T, Power M, et al: Pediatric-based versus adult treatment protocols in young adults (18-40 years) with standard risk acute lymphoblastic leukemia: The BC cancer agency experience. Blood 126: 3770, 2015. 